\title{
Particles Removal from a Moving Tube by Blowing Systems: A CFD Analysis
}

\author{
Giovanni Lombardi, Rubens Curatola \\ Department of Civil and Industrial Engineering, Pisa, Italy \\ Email: lombardi@ing.unipi.it
}

Received October 1, 2012; revised January 28, 2013; accepted February 5, 2013

\begin{abstract}
The use of an air jet to clean the products during the manufacturing is usual in an industrial environment. In this paper the problem of the removal, from the inner of the tube, of the waste arising from the cutting operation by means of an air blowing system is analysed. In order to obtain indications about the real importance of the involved parameters and their effects, a numerical procedure, based on CFD analysis trough the code STAR CCM+, has been settled. The effects of the different parameters were highlighted. The results are congruent with the experience, indicating that the adopted solution is not suitable for the small tubes. Therefore, a different blowing solution is investigated, with the object to improve the cleaning capabilities of the system. The results for the new blowing solution provide a significant improvement of the capability to remove the waste arising from the cutting operation can be expected.
\end{abstract}

Keywords: Fluid Dynamics; Industrial Application; Internal Flow; CFD; Moving Mesh

\section{Introduction}

The use of an air jet to clean the products during the manufacturing is usual in an industrial environment, [1]. In this paper, in particular, the production of glass tubes is considered.

The specific problem is the removal, from the inner of the tube, of the waste arising from the cutting operation. Typically, the tube is transported by a conveyor belt through the various tools. In the considered problem, along the path it meets a sector with some nozzles, which generate air jets to remove the particles remained in the tube after the cutting operation.

The considered problem is affected by several parameters, and it is very difficult a quantitative evaluation of their effects. Therefore, in order to obtain indications about the real importance of the involved parameters and their effects, a numerical procedure, based on CFD analysis, has been settled. For the numerical solution the code STAR CCM+ is used. With the support of the numerical analysis it is possible to highlight the effects of the parameters, and, therefore, to suggest modification to improve the effectiveness of the system.

Finally, a different blowing solution is proposed and analysed.

\section{Overview of the Problem}

The basic scheme consists in a nozzles row, placed below the cutting machine, on one side of the conveyor belt.

The fundamental parameters, see Figure 1, are:

- The internal diameter of the glass tube, $\phi$.

- The length of the tube, L.

- The translation velocity of the tube.

- The gap between the nozzle exhausts and the tube internal side, $w$.

- The number, shape and dimensions of the jet nozzle.

- The total span of the jets, s.

- The supply pressure of the jets, $\Delta \mathrm{p}$ (on respect to the atmospheric pressure).

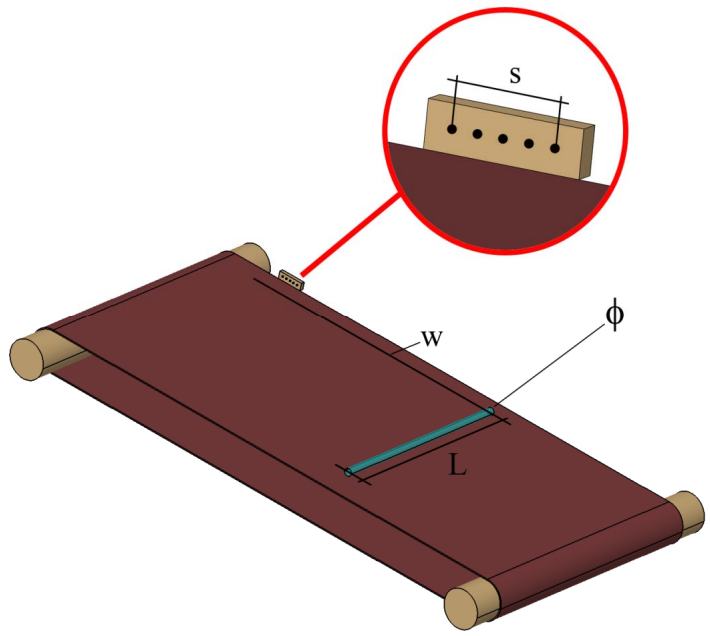

Figure 1. Sketch of the geometrical parameters. 
All the solutions presented in this paper are for a length tube $\mathrm{L}=1570 \mathrm{~mm}$ and a distance between the nozzle exhausts and the tube inner side, $\mathrm{w}$, of $40 \mathrm{~mm}$.

Two different diameters of the tube are considered: the large tube, with internal diameter $\phi=14 \mathrm{~mm}$ (external diameter $16 \mathrm{~mm}$ ) and the small tube, with internal diameter $\phi=6.35 \mathrm{~mm}$ (external diameter $8.15 \mathrm{~mm}$ ). The translation velocity of the tube is fixed by non-aerodynamics considerations; for the large tube it is fixed at 60 $\mathrm{mm} / \mathrm{s}$, while for the small tube it is fixed at $135 \mathrm{~mm} / \mathrm{s}$. Two working pressures $\Delta \mathrm{p}$, are analysed: 101,325 and 303,975 Pa.

A first configuration with a row of five circular jets, with a diameter of $4 \mathrm{~mm}$ and $\mathrm{s}=252 \mathrm{~mm}$, is considered. Its blowing surface is $62.8 \mathrm{~mm}^{2}$.

\section{The CFD Procedure}

\subsection{Conceptual Approach}

The problem under consideration is obviously a nonsteady one, and it is necessary the simulation of the relative movement between two parts: this implies the update of the calculation grid at each time step, [2]. Furthermore, it is necessary to set the time step sufficiently low, in order to have a good representation of the flow time history; a sensitivity analysis to this computational parameter was carried out in a preliminary phase.

The computational domain is divided in two separate domains, one containing the blowing zone, the other containing the tube. The motion is assigned only to the region containing the tube. With this approach, the interface between the two regions is the only part that needs to be update at each time-step.

Finally, because a particularly accurate evaluation of the tangential stresses into the tube is required, a very refined representation with prism layers is created at its internal wall. The sensitivity analysis to grid refinement will be discussed in the following.

The solution gives a complete description of the flow field, at any time step. The analysis of the flow field is clearly interest, and a complete understanding of the problem is available from it. Nevertheless, the amount of data is so large that it is difficult a simple interpretation. Therefore, some "integral" results are derived, in order to have a more clear and immediate idea of the performance of the system.

For this purpose, the tube is divided into 50 sectors, in order to evaluate the distribution along the tube of the quantities of interest. In particular are evaluated the mass flow rate $[\mathrm{Kg} / \mathrm{s}]$, the integral of the shear stress over each sector, indicated with $\tau_{\text {int }}[\mathrm{N}]$, and its value for unit length, referred as $\theta[\mathrm{N} \mathrm{m}]$.

Furthermore, to have an indication of the capabilities of the system to clean the tube, the following integral quantities are considered:

- The mass flow through the end section of the tube, defined as the integral value over the simulation time. It is assumed as index of the available "energy" to remove the particles.

- The integral shear stress over the inner surface of the tube, defined as the integral value over the total inner surface and the simulation time. It is assumed as index of the "traction" force to remove the particles.

- The maximum value of $\tau_{\text {int }}$, over time and space, assumed as index of the capability to "start" the movement of a particle.

\subsection{The Computational Grid}

The surface mesh was generated with ANSA v. 13.01. The volume mesh, generated with $\mathrm{Star} \mathrm{CCM}+$, is composed by polyedrical elements, except the zone close to the wall, represented by prism layers.

The dimensions of the computational domain are reported in Table 1.

The volume grid is characterised by two local refinement zones: one in the ending zone of the tube, the other in the interface zone between the two domains.

Grids up to about 8 millions of cells were analysed in a preliminary phase. The analysis of the results shows that they are essentially stable when the number of grid cells is higher than 3 millions. Therefore, a grid refinement with about 4 millions of cells was settled; the grid parameters chosen for the simulations are shown in Table 2. Some details of the grid are shown in Figure 2.

\subsection{Set-Up of the Numerical Solution}

The numerical simulation can be divided into two phases:

Table 1. Domain dimensions.

\begin{tabular}{ccc}
\hline Length & $2250 \mathrm{~mm}$ & 9 jets row spans \\
Width & $8230 \mathrm{~mm}$ & 5.25 tube lengths \\
Height & $1000 \mathrm{~mm}$ & 62 "large" tube ext. diameters \\
\hline
\end{tabular}

Table 2. Volume mesh parameters.

\begin{tabular}{lc}
\hline Max. Skew & 0.57 \\
\hline Surface cells & $\approx 400.000$ \\
Volume poly cells & $\approx 4 \times 10^{6}$ \\
Poly dens. & 1.1 \\
Poly grow factor & 0.9 \\
Prism layer thickness & $5 \mathrm{~mm}$ \\
Nr of prism layers & 7 \\
Prism layer grow rate & 1.1 \\
\hline
\end{tabular}




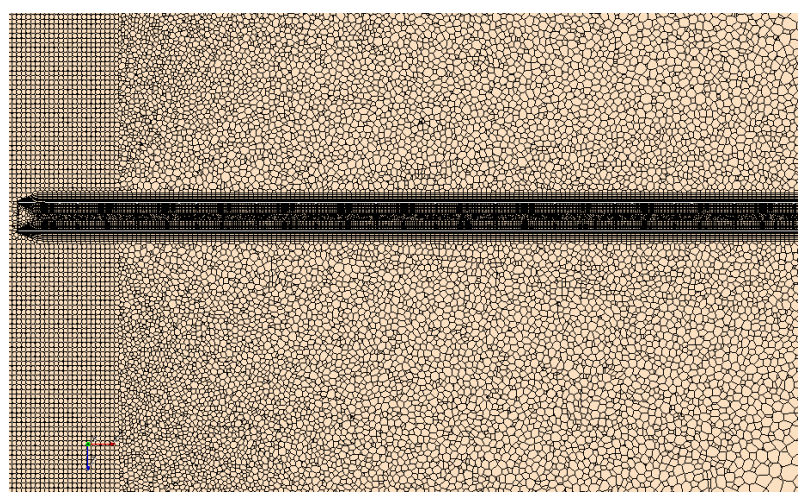

(a)

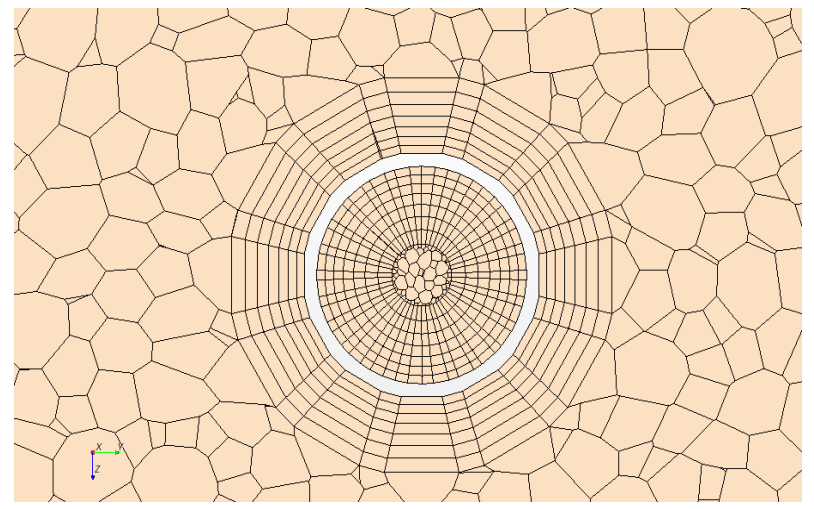

(c)

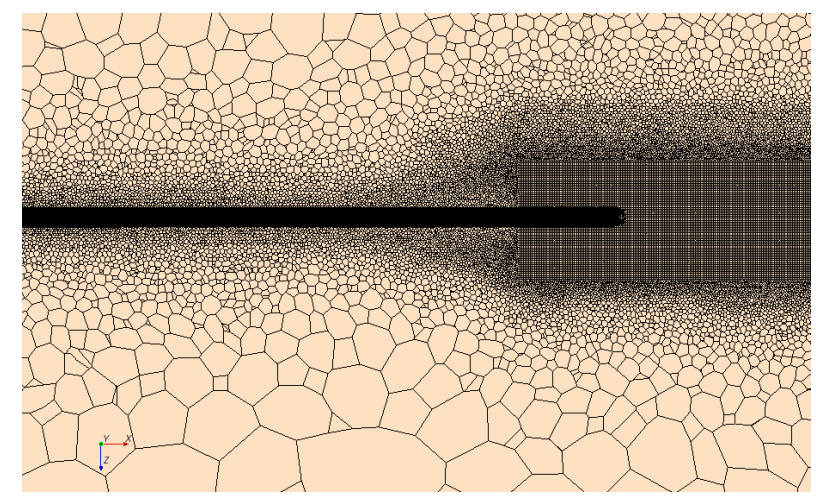

(b)

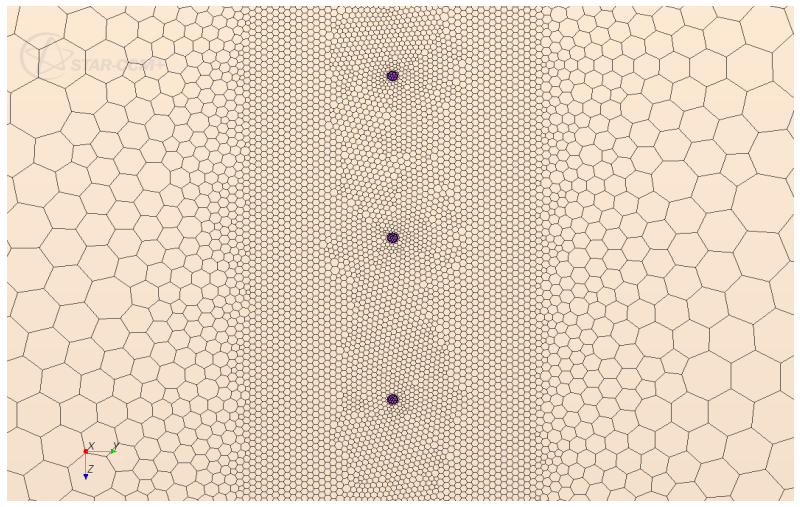

(d)

Figure 2. Details of the volume grid. (a) Inner zone of the tube; (b) Outer zone of the tube; (c) Cross section of the tube; (d) Nozzle inlet.

1) A steady phase to develop the jet flow. The movement of the tube is turned off.

2) The movement of the tube is turned on and the flow regime became unsteady.

The set-up of the calculation is summarised in Table 3.

The energy equation is activated to take into account the different temperature between ambient and walls tube. In particular, the ambient temperature was settled at $28^{\circ} \mathrm{C}$, while the temperature of the tube surfaces was settled at $200^{\circ} \mathrm{C}$.

Several analyses were carried out to choose the time step values to use. The best solution appears to consider a time step variable during the flow evolution, depending on the position of the tube. In particular, a greater value of the time step at the beginning of the simulation, when the air jet is evolving and the tube is far from the nozzles, is acceptable; on the contrary, when the tube is in the jets zone, a very small value of the time step is necessary, depending on the translational speed of the tube.

For each simulation the time histories of the monitored variables and frames of the aerodynamic field at each time step are analysed. Data are then processed in order to obtain the quantities described in the previous section.
Table 3. Computational set-up.

\begin{tabular}{lll}
\hline & Steady phase & Unsteady phase \\
\hline Time & Steady & Implicit unsteady \\
Flow & Coupled flow & Coupled flow \\
Turbulence model & $\mathrm{k}-\varepsilon$ & $\mathrm{k}-\varepsilon$ \\
Wall treatment & Two layer all $\mathrm{y}^{+}$wall & Two layer all $\mathrm{y}^{+}$wall \\
\hline
\end{tabular}

The analysis is considerably challenging in terms of computational costs. For each evaluation the time required is about one week, by using a Linux cluster with 16 SUN Fire X4100, each server with 2 AMD Opteron 285 (Dual Core) processors and 4 GB RAM (64 processes).

\section{Analysis of the Results}

\subsection{The "Large" Tube}

In a first phase the "large" tube, within the described jets set-up, is analysed.

The analysis of the videos, obtained by the frames captured at each time step $(\Delta t=0.005 \mathrm{~s})$, shows that the flow behaviour is similar for both the applied $\Delta \mathrm{p}$, and 
that the air regularly flows into the tube; as an example, the flow fields at four times, at different interference level between the jet and the tube, are shown in Figure 3.

In order to have quantitative indications on the performance, it is interest to analyse the integral data, reported in Table 4. As previously said, the mass flow through the end section of the tube is assumed as index of the available "energy" to remove the particles and, clearly, higher values are preferred. From this point of view, an increase in the applied pressure at the nozzles gives a significant increase, although not proportional to the increment in $\Delta \mathrm{p}$. The integral shear stress over the inner surface of the tube, assumed as the "traction" force to remove the particles, is essentially proportional to $\Delta \mathrm{p}$, such as the maximum $\tau_{\text {int }}$, indicating the capability to "start" the movement of a particle.

Summarising, from data reported in Table 4 it is evident that increasing the applied pressure increase the capabilities of the system to clean the tube.

It is also interest to analyse the variation in time of the mass flow rate at different section along the tube, $\mathrm{x}$, shown in Figure 4. It is evident that, for each of the four considered sections and for both the applied $\Delta \mathrm{p}$, very slight differences in the five periods are present. Therefore, the flow appears oscillatory but well stabilised since the first jet. For the first two sections $(\mathrm{x} / \mathrm{L}=0$ and $\mathrm{x} / \mathrm{L}=$ 0.33 ) a reflux, negative mass flow rate, is present when the tube moves from a jet to the next one. It is evident that a part of the air forced from the jets into the tube is not able to through up the end of the tube, and this means that the involved energy is not completely used to remove the particles. Furthermore, in a reflux zone the particles, previously moved towards the exit of the tube, tends to move in the opposite direction, with a relevant negative effect on the efficacy of the cleaning process.

Another interest aspect is the variation in time of the longitudinal distributions, along the tube, of the value of the shear stress integrated over a cross section, $\theta$, shown in Figures 5 and $\mathbf{6}$ for a period (data are represented any

$0.13 \mathrm{~s}$ ). At time $\mathrm{t}_{1}$ the air start to flow into the tube, and a peak in tangential stress is present at the tube inlet. Increasing time the tangential stress peak moves downstream and the total tangential stress increases. At time $t_{5}$, the peck is close to the end of the tube; at this time the tube is at the end of the jet, and a reflux begin in the tube (part b of the Figures 5 and 6), with a significantly reduction of the tangential stress. From a qualitative point of view, the situation is very similar for both $\Delta \mathrm{p}$, with higher values for the higher $\Delta \mathrm{p}$.

\subsection{The "Small" Tube}

The analysis of the videos, obtained by the frames captured each time step $(\Delta t=0.002 \mathrm{~s})$, shows that the flow behaviour is similar for both the applied $\Delta \mathrm{p}$, but, on respect to the large tube, the air flows along the internal of the tube with less regularity, and a significant reflux occurs when the tube left the jet. As an example, the flow fields at four times, at difference interference level between the jet and the tube, are shown in Figure 7, for the case with $\Delta \mathrm{p}=101325 \mathrm{~Pa}$.

The integral data are reported in Table 5.

The mass flow through the end section increases of only $30 \%$, with a $\Delta p$ that is three times. This behaviour, significantly different from the large tube, indicates that the flow in close to be "blocked" and, therefore, with the small tube the increase of $\Delta p$ do not appears a possible solution to improve the performance of the system, because the energy is far to be completely transferred to the flow.

Table 4. Integral results for the large tube.

\begin{tabular}{lll}
\hline Applied $\Delta \mathrm{p}$ & $101,325 \mathrm{~Pa}$ & $303,975 \mathrm{~Pa}$ \\
\hline Mass flow $[\mathrm{Kg}]$ & 0.0116 & 0.0218 \\
Integral shear stress [N s] & 0.6675 & 2.0165 \\
$\operatorname{Max} \tau_{\text {int }}[\mathrm{N}]$ & 0.0161 & 0.0419 \\
\hline
\end{tabular}

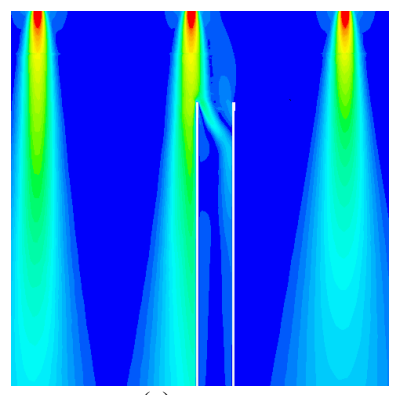

(a)

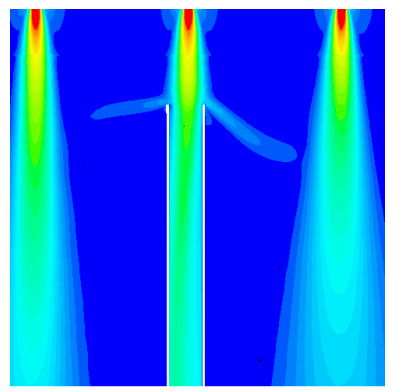

(b)

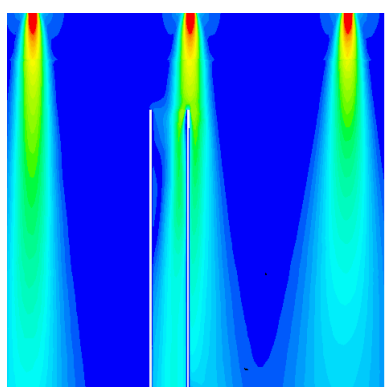

(c)

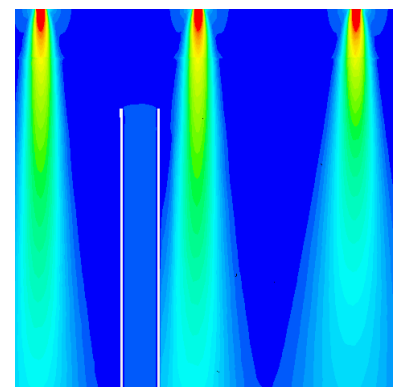

(d)

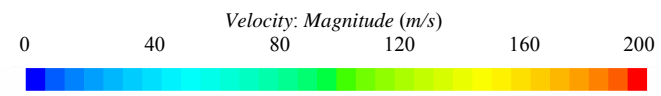

Figure 3. Example of the flow field; large tube, $D p=101,325$ Pa. (a) Initial interference; (b) Complete interference; (c) Final interference; (d) No interference. 

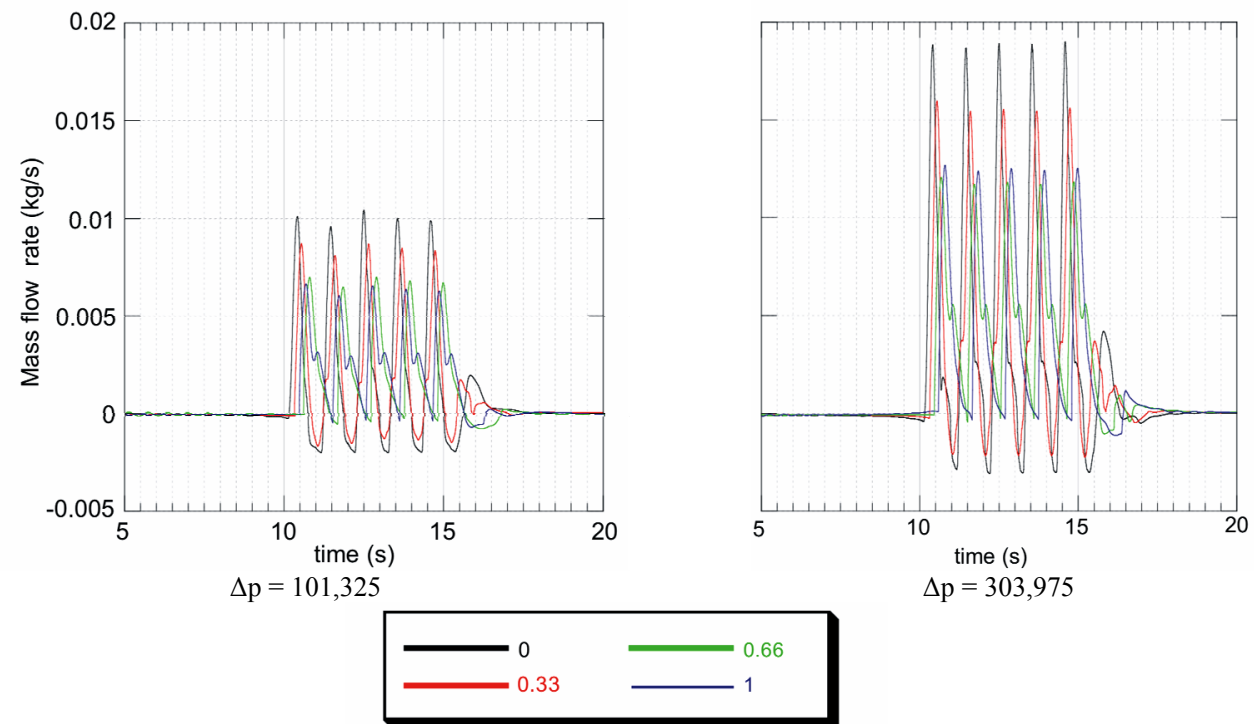

$\mathrm{X} / \mathrm{L}$

Figure 4. Variation in time of the mass flow rate at different section; large tube.

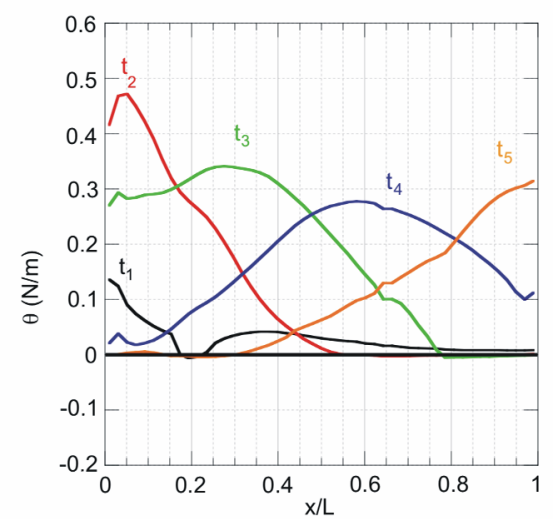

(a)

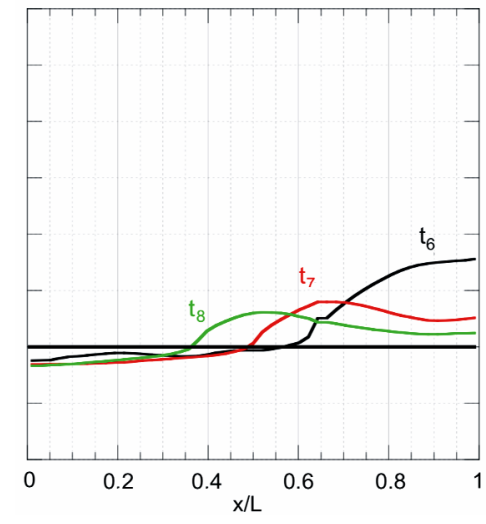

(b)

Figure 5. Longitudinal distribution of the shear stress in a cross section; large tube, $\Delta p=101,325$. (a) No reflux condition; (b) Reflux condition.

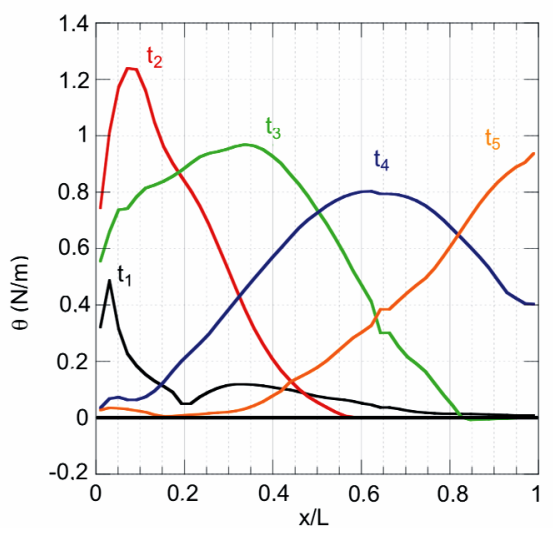

(a)

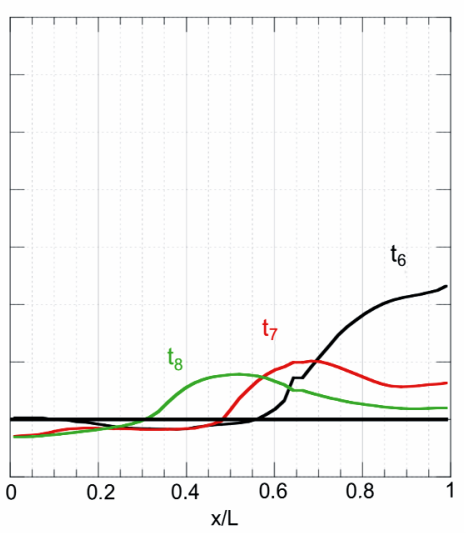

(b)

Figure 6. Longitudinal distribution of the shear stress in a cross section; large tube, $\Delta p=303,975$. (a) No reflux condition; (b) Reflux condition. 


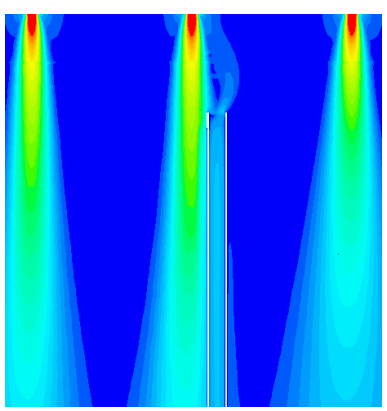

(a)

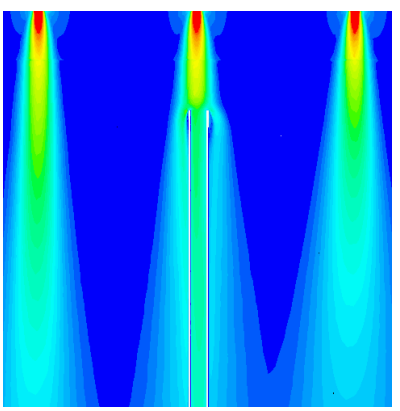

(b)

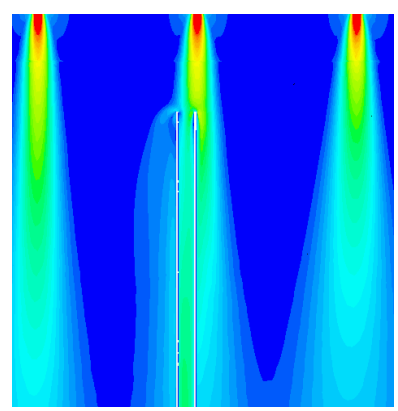

(c)

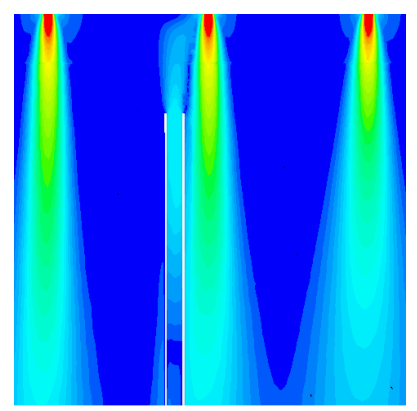

(d)

0

Velocity: Magnitude $(\mathrm{m} / \mathrm{s})$

80

120

160 200

Figure 7. Example of the flow field; small tube. (a) Initial interference; (b) Complete interference; (c) Final interference; (d) No interference.

Table 5. Integral results for the small tube.

\begin{tabular}{lll}
\hline Applied $\Delta \mathrm{p}$ & $101,325 \mathrm{~Pa}$ & $303,975 \mathrm{~Pa}$ \\
\hline Mass flow $[\mathrm{Kg}]$ & 0.0019 & 0.0025 \\
Integral shear stress [N s] & 0.0996 & 0.2848 \\
$\operatorname{Max} \tau_{\text {int }}[\mathrm{N}]$ & 0.0124 & 0.0260 \\
\hline
\end{tabular}

On the contrary, the integral shear stress along the inner surface of the tube, assumed as "traction" force to remove the particles, appears essentially proportional to $\Delta \mathrm{p}$, but the maximum $\tau_{\text {int }}$, indicating the capability to "start" the movement of a particle, is far to be proportional to $\Delta \mathrm{p}$, as occurs for the large tube. From data reported in Table 5 it is evident that, for the small tube, increasing the applied pressure is not a suitable method to increase the capabilities of the system to clean the tube. The variation in time of the mass flow rate at different section along the tube, $x$, are shown in Figure 8.

For each of the four considered sections and for both the applied $\Delta \mathrm{p}$, significant differences in the five periods are present, indicating that the flow is not well stated. Furthermore, the reflux appears really important, significantly higher than for the large tube. Therefore, it is evident that the cleaning capabilities for the small tube are significantly reduced.

The variation in time of the longitudinal distributions, along the tube, of the value of the shear stress integrated over a cross section, are shown in Figures $\mathbf{9}$ and $\mathbf{1 0}$ for a period (data are represented any $0.058 \mathrm{~s}$ ). The behaviour is similar to the large tube, but the values are significantly lower and the reflux, that appears significantly higher, occurs at any time.

In conclusion, the results confirm the experience, indicating that the adopted solution appears suitable for the large tube, but not for the small one.
Therefore, a different blowing solution was investigated, with the object to improve the cleaning capabilities of the system, particularly for the small tube. The results will be described in the next section.

\subsection{A Proposed Different Blowing System}

As previously shown, in the original solution the flow has a pulsed trend, due to the non-continuity of the air jet. This leads to a phase with a reverse flow, with a significant reduction of the efficacy of the system.

To improve the situation, the proposal is to replace the nozzles with a single blade, as shown in Figure 11. The analysed configuration has a span $\mathrm{s}=150 \mathrm{~mm}$ and height $\mathrm{h}=0.5 \mathrm{~mm}$. Therefore, the blowing surface is $75 \mathrm{~mm}^{2}$, slightly higher $(19.4 \%)$ on respect the original solution.

The study is carried out for the small tube (the critical one), at the higher $\Delta \mathrm{p}, 303,975 \mathrm{~Pa}$.

The analysis of the videos, obtained by the frames captured each time step $(\Delta t=0.005 \mathrm{~s})$, shows a completely different flow, as can be seen from Figure 12. It is evident that, with this blowing system, the flow appears completely regular during the entire blowing phase.

The integral data are reported in Table 6. The increase in the system efficacy appears evident. The mass flow through the end section of the tube, assumed as index of the available "energy" to remove the particles, shows a significant increase, about $65 \%$, while the integral shear stress along the inner surface of the tube, assumed as "traction" force to remove the particles, increases of $68 \%$. On the other side, the maximum $\tau_{\text {int }}$, assumed as index of the capability to "start" the movement of a particle is reduced. This because the flow is now regular, while the high peak in the basic solution appears related to transitional phase of the flow.

The variation in time of the mass flow rate at different section along the tube, $x$, are shown in Figure 13. It is clear that the behaviour is now completely different. The 


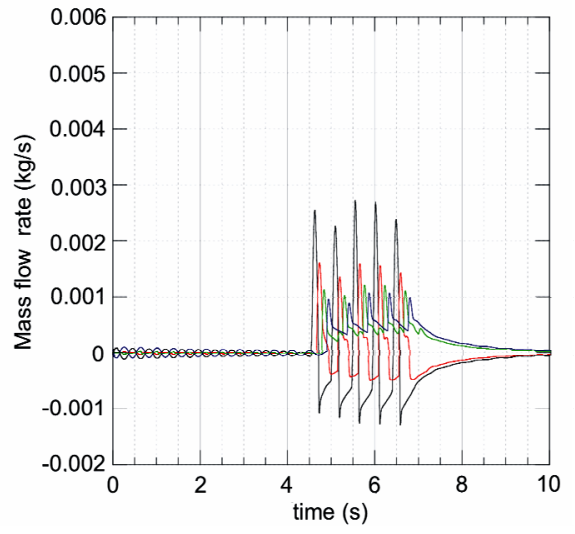

$\Delta \mathrm{p}=101,325$

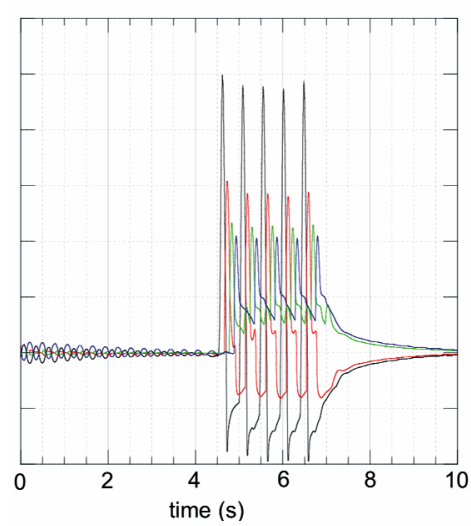

$\Delta \mathrm{p}=303,975$

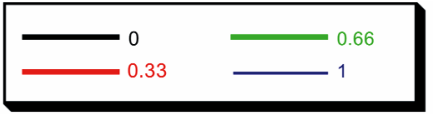

$\mathrm{x} / \mathrm{L}$

Figure 8. Variation in time of the mass flow rate at different section; large tube.

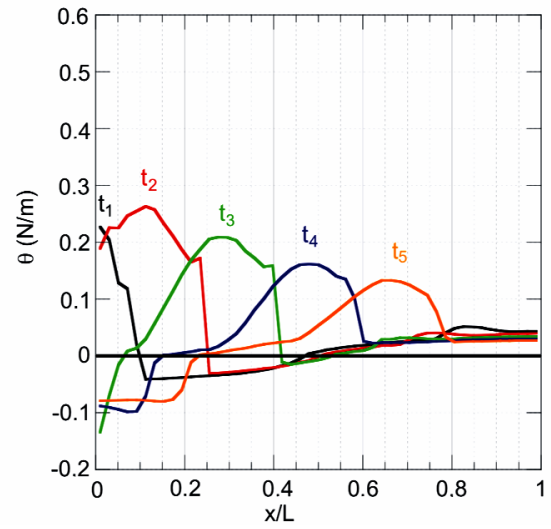

(a)

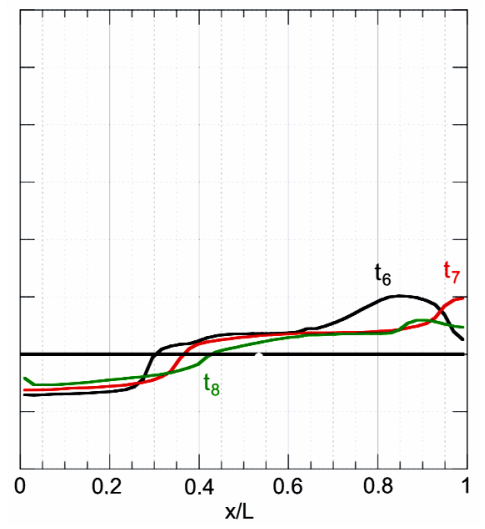

(b)

Figure 9. Longitudinal distribution of the shear stress in a cross section; small tube, $\Delta p=101,325$. (a) Initial phase of the period; (b) Final phase of the period.

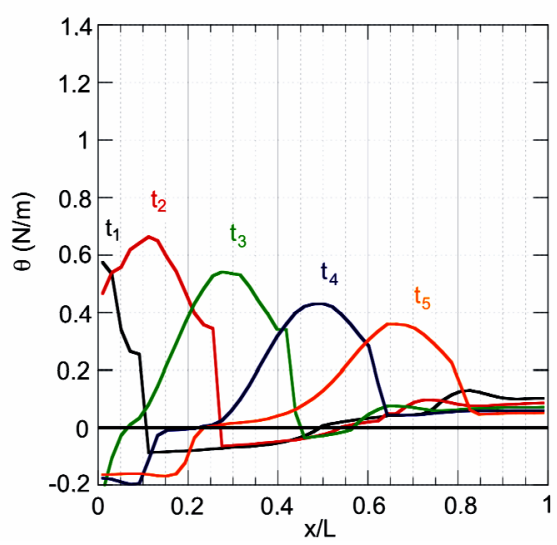

(a)

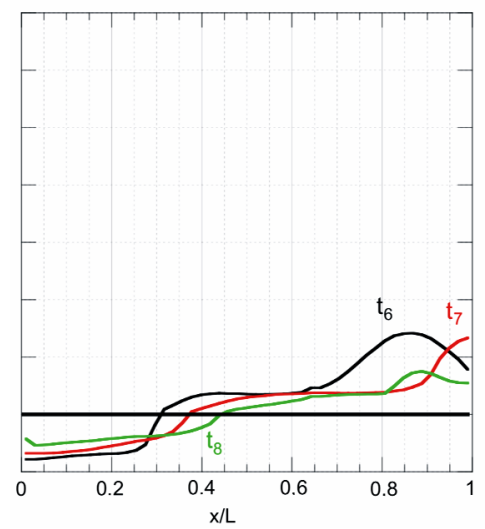

(b)

Figure 10. Longitudinal distribution of the shear stress in a cross section; small tube, $\Delta p=303,975$. (a) Initial phase of the period; (b) Final phase of the period. 


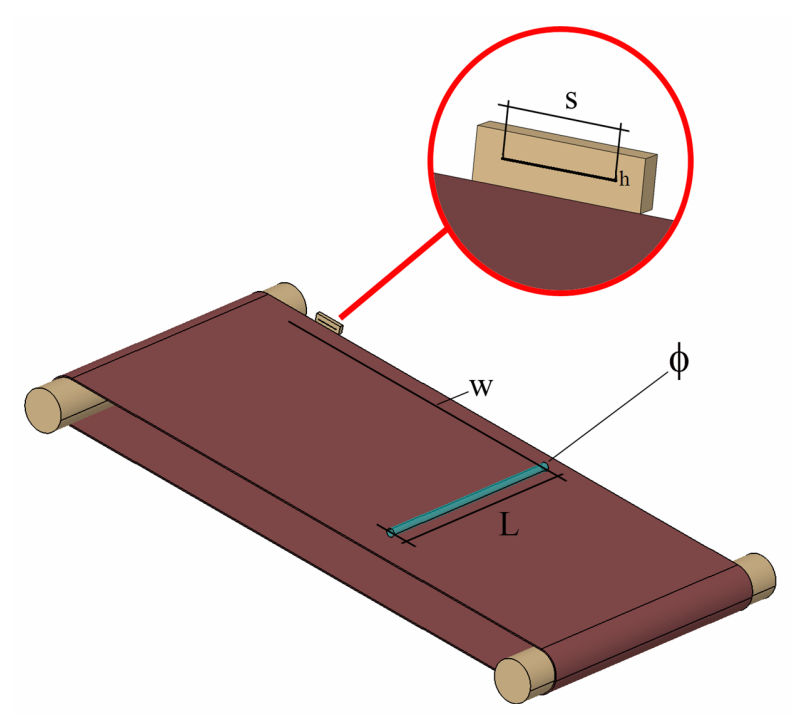

Figure 11. Sketch of the geometrical parameters.

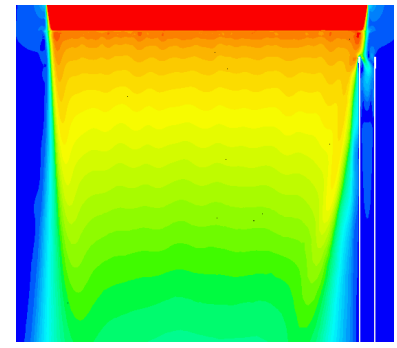

(a)

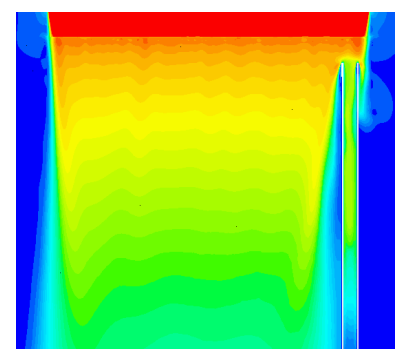

(c)

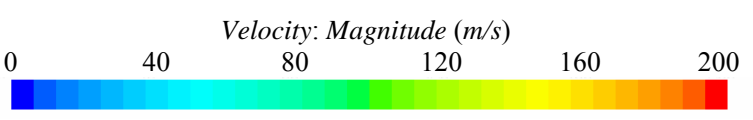

Figure 12. Example of the flow field, blade solution. (a) No interference; (b) Initial interference; (c) Complete interference; (d) Regime interference.

flow appears almost constant up to the end of the interference between the tube and the blowing (the interference time, at the considered translational velocity of the tube, is $1.1 \mathrm{~s}$ ). Furthermore, the reflux appears only behind the end of the blowing phase.

To highlight the different behaviour of the two solutions, in Figure 14 the time history of the mass flow rate at the outlet section are shown. It is evident that the blade solution shows a higher continuity in the flow, with an
Table 6. Integral results for the two proposed blowing solutions.

\begin{tabular}{lll}
\hline Scheme & Basic & Blade \\
\hline Mass flow $[\mathrm{Kg}]$ & 0.0025 & 0.0041 \\
Integral shear stress $[\mathrm{N} \mathrm{s}]$ & 0.2848 & 0.4785 \\
$\operatorname{Max} \tau_{\text {int }}[\mathrm{N}]$ & 0.0260 & 0.0202 \\
\hline
\end{tabular}

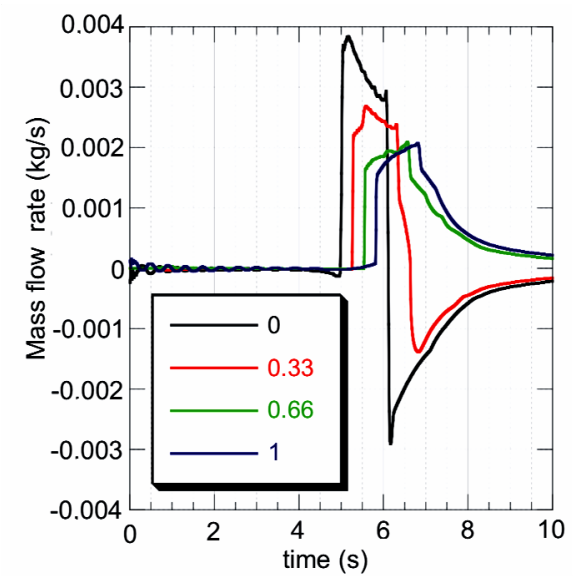

Figure 13. Variation in time of the mass flow rate at different section; blade blowing.

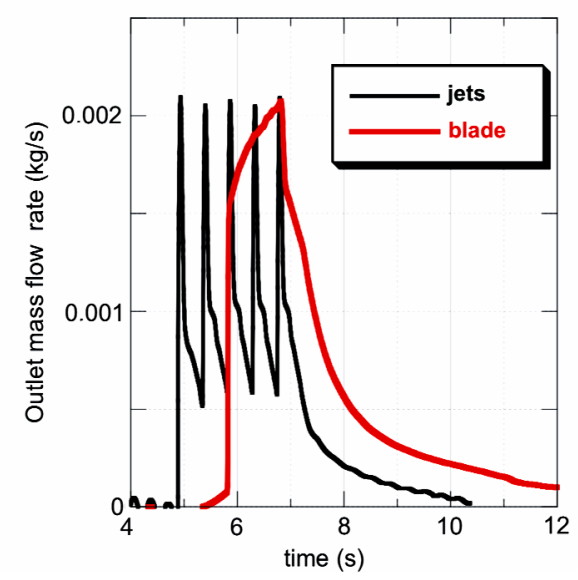

Figure 14. Outlet mass flow rate for the two blowing solutions.

expected significant increase oh the cleaning capabilities.

Finally, the variation in time of the longitudinal distribution, along the tube, of the value of the shear stress integrated over a cross section is shown in Figure 15 (data are represented any $0.15 \mathrm{~s}$, therefore the total time is significantly higher than in the corresponding Figure 10). The behaviour is completely different from that of the jets solution. The tangential stress appears almost constant over all the tube, and no reflux appears up to final time, when the tube is completely out from the blow. 

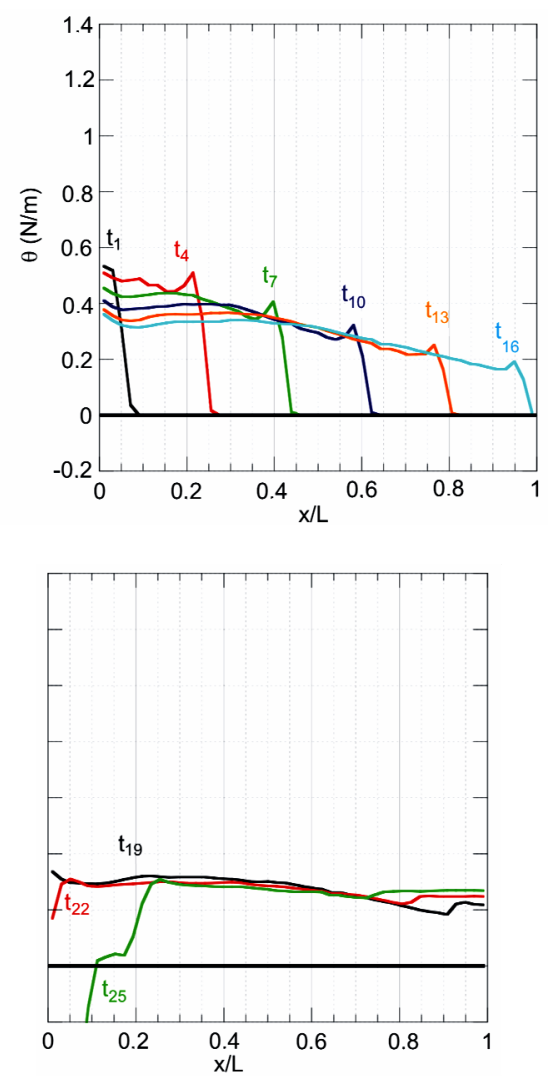

Figure 15. Longitudinal distribution of the shear stress in a cross section; blade solution.

In conclusion, all the results indicate that the cleaning capabilities would be significantly improved with the blade blowing system.

\section{Conclusions}

The problem of the removal, from the inner of the tube, of the waste arising from the cut operation by means of an air blowing system was analysed. In order to obtain indications about the real importance of the involved parameters and their effects, a numerical procedure, based on CFD analysis trough the code STAR CCM+, has been settled.

With the support of the numerical analysis the effects of the parameters were highlighted. The results confirm the experience, indicating that the adopted solution appears suitable for the large tube, but not for the small one. Therefore, a different blowing solution was investigated, with the object to improve the cleaning capabilities of the system.

The results indicate that, in order to remove the waste arising from the cutting operation, a significant improvement of the capability can be expected with the new blowing solution.

\section{REFERENCES}

[1] A. Guha, R. M. Barron and R. Balachandar, "An Experimental and Numerical Study of Water Jet Cleaning Process," Journal of Materials Processing Technology, Vol. 211, No. 4, 2011, pp. 610-618.

[2] M. M. Pror, “Automated Moving Mesh Techniques and Re-Meshing Strategies in CFD Applications Using Morphing and Rigid Motions," CRS4 Technical Report, 2012. 